\title{
Motivación: un recorrido histórico y teórico de los principales
} marcos conceptuales

Motivation: a historical and theoretical trajectory of the main conceptual frameworks

\author{
Recepción del artículo: 07-11-2020 ～～Aceptación del artículo: 21-12-2020
}

Matías, Serafini

Universidad de Buenos Aires, Facultad de Medicina, Instituto de investigaciones Médicas

A. Lanari.

Consejo Nacional de Investigaciones Científicas

y Técnicas, Universidad de Buenos Aires, Instituto de Investigaciones Médicas (IDIM),

Laboratorio de Psicología Experimental y

Aplicada.

Universidad Abierta Interamericana, Centro de

Altos Estudios en Ciencias Humanas y de la

Salud, Facultad de Psicología y Relaciones

Humanas, Argentina matiasserafini@gmail.com

iD https://orcid.org/0000-0002-1078-7834

Lucas, Cuenya

Universidad de Buenos Aires, Facultad de Medicina, Instituto de investigaciones Médicas

A. Lanari.

Consejo Nacional de Investigaciones Científicas y Técnicas, Universidad de Buenos Aires, Instituto de Investigaciones Médicas (IDIM),

Laboratorio de Psicología Experimental y

Aplicada.

Universidad Abierta Interamericana, Centro de Altos Estudios en Ciencias Humanas y de la

Salud, Facultad de Psicología y Relaciones

Humanas, Argentina

Lucascuenya@gmail.com

https://orcid.org/0000-0003-2650-8374

Para referenciar este artículo:

Serafini, M. y Cuenya, L. (2020). Motivación: un recorrido histórico y teórico de los principales marcos conceptuales. Revista ConCiencia EPG, 5(2), 15-44. https://doi.org/10.32654/CONCIENCIAEP

\section{Resumen}

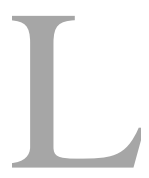

a literatura sobre estudios motivacionales presenta una enrevesada yuxtaposición de definiciones provenientes de marcos teóricos disímiles. Un inconveniente que surge al revisar esta literatura es la polisemia que hay en torno al constructo. En un sentido general la motivación se relaciona con procesos básicos que proporcionan energía y dirección a la conducta. La energía implica que el comportamiento tiene fortaleza, intensidad $\mathrm{y}$ persistencia. La dirección refiere a que la conducta tiene un propósito y está orientada a una meta en particular. Se puede esbozar una división en torno al origen de la energía (i.e., interna vs externa) o la dirección del comportamiento (i.e., intrínseco vs extrínseco). La motivación no debe entenderse como una serie de categorías aisladas u opuestas, sino como un sistema multicomponente que dinamiza y orienta la conducta hacia el logro de una meta. En esta revisión se recorren los principales marcos conceptuales de las ciencias del comportamiento a partir de los que se desprenden términos motivacionales, desde la psicología experimental en modelos animales y los últimos hallazgos neurocientíficos hasta los trabajos sobre psicología sociocognitiva en humanos, con el objetivo de entablar lazos entre los estudios básicos y aplicados.

Palabras Clave: Fuentes de Motivación; Motivación de Incentivo; Motivación Intrínseca, Motivación Extrínseca; Liking; Wanting. 
Abstract

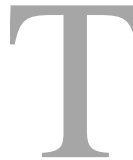

he literature on motivational studies presents a convoluted juxtaposition of definitions from dissimilar theoretical frameworks.

The polysemy surrounding the construct is a drawback that arises when the literature on motivation is reviewed. In a general sense, motivation is related to basic processes that provide energy and direction to behavior. Energy implies that behavior has strength, intensity, and persistence. Direction implies that the behavior has a purpose and is oriented to a particular goal. A division can be outlined around the origin of energy (i.e., internal vs external) or the direction of behavior (i.e., intrinsic vs extrinsic). Motivation should not be understood as a series of isolated or opposing categories, but as a multicomponent system that energizes and directs behavior towards the achievement of a goal. This review covers the main conceptual frameworks of the behavioral sciences from which motivational terms emerge, from experimental psychology in animal models and the latest neuroscientific finding to studies on sociocognitive psychology in humans, with the aim of establishing ties between basic and applied research.

Key Words: Sources of Motivation; Incentive Motivation; Intrinsic Motivation, Extrinsic Motivation; Liking; Wanting.
Introducción

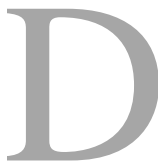

urante las últimas décadas, el interés por la motivación ha resurgido como un tópico crucial para las ciencias del comportamiento y la neurociencia en el intento de comprender cómo los organismos presentan respuestas complejamente organizadas. Los términos que remiten a conceptos o modelos motivacionales se encuentran presentes de extremo a extremo en las ciencias de la conducta, desde tópicos sobre neurociencia (e.g., Berridge, 1996; Berridge, 2009a; Berridge, 2009b; Berridge et al., 2009; Castro \& Berridge, 2014a; Smith \& Berridge, 2005), desórdenes psicopatológicos como los trastornos de la conducta alimentaria y las adicciones (e.g., Flagel \& Robinson, 2017; Guarda, Schreyer, Boersma, Tamashiro, \& Moran, 2015; Holsen et al, 2012; Robinson, Yager, Cogan, \& Saunders, 2014; Saunders, Yager \& Robinson, 2013; Shaham, Shalev, Lu, de Wit, \& Stewart, 2002) hasta áreas de investigación aplicada de gran interés actual como el aprendizaje escolar (e.g., Autio, 2011; Lin, McKeachie, \& Kim, 2003; Meyers, Pignault, \& Houssemand, 2013). Sin embargo, el interés por la motivación y la presencia transversal de los conceptos motivacionales no se ha acompañado de una sistematización teórica que permita asegurar un buen nivel de rigurosidad en el empleo de dichos términos. De este modo, la literatura sobre estudios motivacionales presenta una enrevesada yuxtaposición de definiciones provenientes de marcos teóricos disímiles. Un gran inconveniente que surge al revisar la literatura sobre la motivación es la polisemia que hay en torno 
al constructo. Esta noción, a pesar de encontrarse en las teorías psicológicas generales desde hace más de un siglo, tiene aún hoy una definición poco consensuada. El concepto resulta tan amplio en sus posibles acepciones que se torna necesario hacer una revisión de los aportes psicológicos y neuroconductuales que posibilitaron los desgranamientos conceptuales que en la actualidad se le confieren.

En términos generales, la motivación es un concepto teórico cuyo sentido etimológico estricto refiere a la palabra motivo, el cual proviene del latín motivus (i.e., relativo al movimiento; Real Academia Española, 2019). Al parecer, estar motivado significa ser movido a hacer algo o en alguna dirección, y distintos términos se utilizan, coloquial o académicamente, para hacer referencia a esa fuerza: impulsos, pulsiones, anhelos, apetitos, deseos, necesidades o intereses, entre otros. De esta forma, empleamos términos motivacionales para hacer referencias a estados internos. Apelamos a los términos motivacionales para que nos auxilien al momento de comprender los cambios del comportamiento en un individuo, o las diferencias entre individuos. La motivación es uno de los conceptos que nos permite explicar -o al menos intentarlo- uno de los hechos menos controvertibles en relación al comportamiento: la enorme variabilidad de respuesta interindividual ante idénticas condiciones ambientales. Por tanto, la motivación puede entenderse como una variable intermedia entre los factores antecedentes, tanto del ambiente como del propio organismo, y la respuesta o conjunto de respuestas que el organismo expresa.
Sin embargo, decir tan sólo que la motivación es un estado interno resulta una definición demasiado difusa. Aguado (2005) aporta una definición general que permite enmarcar los mecanismos conductuales y psicobiológicos que son abordados por una porción mayoritaria de la literatura. Este autor define a la motivación como el conjunto de mecanismos que energizan, organizan e inician la conducta mientras la orientan a la consecución de metas o recompensas relevantes. Es importante señalar que, desde esta perspectiva, el comportamiento motivado no es errático, sino dirigido y organizado con una función primordial: maximizar las chances de obtener consecuencias deseadas o convenientes y minimizar la probabilidad de obtener consecuencias indeseadas 0 no convenientes para el organismo. Este comportamiento puede ser o no voluntario y planificado, puede tener o no mediación consciente, pero siempre estará ligado a una meta.

Otro problema que surge al examinar la literatura sobre estudios motivacionales, al menos en medios académicos regionales, reside en la alta popularidad de teorías con escasa posibilidad de contrastabilidad empírica, lo que no permite sujetar sus consecuencias observacionales al método científico. Un ejemplo de esto es la teoría psicoanalítica, la que intenta dar cuenta de los comportamientos aparentemente sin sentido - síntomas - de los sujetos bajo análisis, lo que la convierte en una teoría fuertemente interesada en la motivación del comportamiento. Al menos en su planteo original, Freud (1915) formuló el 
concepto de pulsión (trieb) - tributario del concepto de instinto - con el fin de explicar el comportamiento de los sujetos, en particular en el contexto clínico. Las pulsiones, entonces, serían impulsos psíquicos que llevarían al individuo a actuar ante una tensión interna, reduciéndolo a través de un objeto. El objeto en el comportamiento humano funcionaría como un motivador, pero a diferencia de los animales no humanos, no presentaría una predeterminación innata, sino que sería el resultado de la interacción singular del sujeto con su medio a lo largo del recorrido de su ontogenia. Las pulsiones se subdividían, según Freud, en distintos tipos en función del objeto y la dirección hacia la cual impulsan al sujeto, siendo las más renombradas la pulsión de Eros o de vida, y la pulsión de Tánatos o de muerte. En este sentido, la teoría psicoanalítica adoleció de un inconveniente común en su época: el carácter tautológico de enunciados que se pretendían explicativos. La nominación de una motivación que empuja el comportamiento en una dirección no constituye en sí mismo una explicación de los mecanismos que gobiernan los comportamientos.

Sería erróneo asumir que todas las teorías psicológicas motivacionales no psicodinámicas que cuentan con extensa popularidad, también cuentan con tan extensa validación empírica. Este es el caso de la teoría motivacional de Maslow (1943; 1954), la que propone que las personas presentan cinco tipos de necesidades jerárquicas. En la medida que se satisface un nivel de necesidad, se activa un anhelo por satisfacer la necesidad subsiguiente. Según este modelo, las necesidades más básicas serían fisiológicas (e.g., alimentación). Cuando las necesidades fisiológicas están en gran parte satisfechas, surgen necesidades orientadas hacia la seguridad personal, el orden, la estabilidad y la protección. Posteriormente se desplegarían necesidades sociales orientadas a la búsqueda del amor, el afecto y la pertenencia a un grupo social, para situar en un siguiente escalafón a las necesidades orientadas hacia la búsqueda de la autoestima, el estatus o la reputación. Por último, las necesidades de autorrealización son aquellas que tienen el nivel jerárquico más alto. En un inicio, la teoría recibió fuertes críticas por carecer de un sustento empírico adecuado (Wahba \& Bridwell, 1976). Si bien estudios psicométricos actuales se centraron en remediar esto aportando datos a favor de algunas de las consecuencias observacionales de la jerarquía de necesidades, la evidencia no fue lo suficientemente robusta (e.g., Collins, Lanham, \& Sigford, 2000; Taormina \& Gao, 2013; Winston, Maher \& Easvaradoss, 2017).

El objetivo de esta revisión es clarificar el mapa conceptual de las principales teorías desde las que se desprenden conceptos motivacionales y facilitar un nexo entre la investigación básica y aplicada. Se repasarán preponderantemente estudios provenientes de la psicología experimental en modelos animales y las neurociencias, ya que han aportado un cuerpo de conocimientos y modelos conceptuales robusto en el intento de generar marcos explicativos sobre los mecanismos motivacionales. Una objeción a la 
utilización de modelos animales en psicología señala que estos no permiten abordar la complejidad del comportamiento humano, lo que constituye una crítica válida. Sin embargo, un modelo es una representación a escala reducida de un fenómeno conductual, cuyo objetivo es proveer al investigador de ventajas metodológicas como la manipulación de variables independientes, el registro preciso de variables dependientes y un amplio control experimental de variables extrañas.

\section{Fuentes de motivación}

La motivación se relaciona con procesos básicos que proporcionan energía y dirección a la conducta. La energía implica que el comportamiento tiene fortaleza, intensidad y persistencia. La dirección refiere a que la conducta tiene un propósito y está orientada a una meta en particular. La conducta motivada no sucede de manera espontánea, sino que puede ser inducida tanto por motivos internos como por incentivos ambientales. La única forma de comprender la acción de la motivación sobre el comportamiento radica en observar las relaciones entre los factores internos y externos al organismo. Cuando se considera que la motivación es aquel impulso que empuja al organismo en cierta dirección, se hace referencia a su aspecto energético. De tal forma, se puede esbozar una primera división en torno al origen de la fuerza o energía: interna vs externa. Tal diferenciación pretende aportar un ordenamiento conceptual, sin proponer una clasificación taxativa de las fuentes motivacionales, dado que, por lo general, un mismo comportamiento se encuentra traccionado por múltiples fuentes que interactúan entre sí.

\section{Fuentes de motivación interna}

Se han propuesto distintos términos que podrían entenderse como fuentes internas de la motivación, siendo los más importantes los impulsos y las necesidades fisiológicas. Las necesidades son condiciones dentro del individuo que resultan esenciales para la preservación de la vida. El hambre y la sed ejemplifican dos estados motivacionales que surgen a partir de un déficit fisiológico. Estos estados activan y orientan la conducta de búsqueda e ingesta que llevará a recuperar el equilibrio orgánico.

Un concepto estrechamente vinculado al impulso o necesidad es el de homeostasis, término inicialmente propuesto por Cannon (1932, en Cooper, 2008). La homeostasis es entendida como el estado de equilibrio dinámico o el conjunto de mecanismos por los que un organismo tiende a alcanzar la estabilidad de su medio interno. La motivación homeostática depende de un mecanismo de retroalimentación negativa que compara el estado actual del organismo con un setpoint o estado meta a ser alcanzado para mantener los niveles fisiológicos dentro de parámetros óptimos en relación a las condiciones biológicamente deseables para el organismo. Si estos estados no coinciden, se corrigen los errores hasta que ambos sean equivalentes. Los componentes de este tipo de mecanismos son similares a los de un termostato. En el caso de la temperatura, un sensor detecta el estado actual, y si hay discrepancias al compararlo con el estado 
meta o setpoint se iniciarán mecanismos de corrección (Berridge, 2004).

Otro concepto clave para referirse a los impulsos o necesidades de orden interno es el de drive, el que alude al estímulo interno persistente que proviene de una necesidad fisiológica producto de un desequilibrio homeostático. Este concepto fue propuesto inicialmente por Hull (1943) como componente determinante del aprendizaje. Este autor planteó el modelo de secuencia necesidad-drivecomportamiento, modelo según el cual los impulsos son biológicamente inevitables y ocurren de modo cíclico y a intervalos periódicos. En el caso del hambre, por ejemplo, el patrón cíclico que presenta el surgimiento y la disminución del drive comienza con una privación fisiológica prolongada que causa una necesidad corporal. Luego, esta necesidad se intensifica y da origen al impulso o tensión, el que provee de dirección y energía a la conducta motivada para la obtención de los estímulos que permiten reducirlo y retornar al organismo a un estado homeostático (e.g., búsqueda de alimentos y respuestas consumatorias). De esta manera, la reducción del drive permite alcanzar al organismo el estado de saciedad hasta que el ciclo vuelva a comenzar por una nueva privación fisiológica.

Otros autores han tomado el concepto de drive para abordar su interacción con los estímulos ambientales. Por ejemplo, Lorenz y Leyhausen (1973) propusieron una analogía hidráulica para comprender dicha interacción. Según este modelo, la motivación aumenta internamente y opera como la presión de un depósito de agua que crece hasta que irrumpe a través de una salida. La fuerza interna interactúa con la fuerza motivacional producto de la presentación de un estímulo ambiental. Los estímulos evolutivamente relevantes para el individuo (e.g., un alimento con alta contribución energética) producirán una fuerza motivacional externa mayor que estímulos con menos relevancia. En este modelo, la interacción entre el drive (impulso interno) y los estímulos ambientales depende de la intensidad de ambas partes. Si el drive es bajo, es necesario un fuerte estímulo externo para desencadenar la conducta motivada. Por ejemplo, en un estado de saciedad un animal no tendrá las claves interoceptivas del hambre, por lo que su nivel de drive para la obtención de alimentos será mínimo. Sin embargo, si se encuentra con un alimento altamente apetitivo $\mathrm{y}$ energético, este estímulo puede incrementar externamente la motivación e incitar a la conducta de ingesta. El caso contrario lo representa una situación en la cual el drive es muy alto pero el estímulo ambiental muy leve. Por ejemplo, un animal extremadamente hambriento que se encuentra con un alimento desagradable. La conducta de ingesta en este caso puede incitarse y sostenerse a pesar de que el estímulo ambiental sea poco atractivo, puesto que el drive es lo suficientemente elevado.

\section{Fuentes de motivación externa}

\section{Reforzadores e incentivos}

Las principales fuentes externas de la motivación son los reforzadores e 
incentivos. Por reforzador se entiende a aquellos estímulos cuya ocurrencia aumentan la probabilidad de una respuesta instrumental a la que sigue (Mackintosh, 1988); mientras que los incentivos son a aquellos estímulos que a partir de experiencias previas adquirieron la capacidad de anticipar las propiedades del reforzador $\mathrm{y}$, por tal capacidad, forman expectativas y empujan la conducta generando lo que se denomina motivación de incentivo (Beck, 2000; Logan, 1968).

Los incentivos y los reforzadores pueden aparentar cumplir una misma función, sin embargo, intervienen en la motivación de diferente manera. Un modelo que plantea esta distinción es el de Baldwin y Baldwin (1986, en Reeve, 2003) cuyos componentes básicos son las señales de una situación (S), la Respuesta conductual (R) y la Consecuencia (C). Se puede representar de la siguiente forma $\mathrm{S}: \mathrm{R} \longrightarrow \mathrm{C}$. Los dos puntos entre $S$ y $R$ muestran que la señal situacional establece el despliegue de la respuesta conductual pero no su causa. La flecha entre $\mathrm{R}$ y $\mathrm{C}$ muestra que la respuesta conductual da origen a una consecuencia. De esta forma, el incentivo será un suceso ambiental que atrae o repele a un individuo respecto de una acción particular. Para este modelo los incentivos siempre preceden a la conducta (S:R) formando expectativas y anticipando las consecuencias gratificantes o aversivas. Según este modelo los incentivos no causan conducta, intervienen en la posibilidad de que se vigorice o debilite el inicio de una respuesta conductual. En cambio, el refuerzo implica consecuencia contingente a una conducta (RQC) $\mathrm{y}$, como tal, es posterior al comportamiento, aumentando o disminuyendo su persistencia (i.e., la probabilidad de ocurrencia de la conducta; Baldwin \& Baldwin, 1986, en Reeve, 2003).

El proceso o mecanismo básico por el cual los incentivos adquieren propiedades motivacionales se denomina aprendizaje pavloviano. En el condicionamiento clásico o pavloviano, la presentación de una señal (i.e., estímulo condicionado, EC) está asociado con la presentación de un reforzador (i.e., estímulo incondicionado, EI), y con repetidos emparejamientos el EC incita una respuesta condicionada (RC) similar a la respuesta incondicionada (RI) desplegada por el EI (Domjan, 2010). De esta forma los EC adquieren tres propiedades fundamentales. En primer lugar, alcanzan la capacidad de generar un comportamiento de seguimiento de señal (e.g., Flagel, Watson, Akil, \& Robinson, 2008; Peterson, Ackilt, Frommer, \& Hearst, 1972). En segundo lugar, pueden potenciar conductas instrumentales por efecto de transferencia de condicionamiento clásico a instrumental (e.g., Dickinson, Smith \& Mirenowicz, 2000; Lovibond, 1983; Wyvell \& Berridge, 2000). En tercer lugar, logran actuar como reforzadores condicionados o secundarios en el aprendizaje de nuevas conductas instrumentales (e.g., Di Ciano \& Everitt, 2004; Williams \& Dunn, 1991).

El seguimiento de señal es un fenómeno conductual en el que una clave adquiere propiedades de incentivo que predicen el estímulo incondicionado. De esta manera, los individuos despliegan patrones conductuales dirigidos a la clave (Ver Flagel, Akil, \& Robinson, 2009). Lo curioso de esta conducta es que no hay una relación de contingencia entre la respuesta 
de acercamiento a la clave y la obtención del reforzador. Por tales motivos, uno podría preguntarse: ¿qué es lo que motiva el acercamiento a la señal?.

El automoldeamiento es un ejemplo de cómo se direccionan los comportamientos hacia el estímulo predictor del reforzador. La configuración de la RC va a depender de la especie y de la naturaleza de los EC y los EI. La literatura previa mostró que existe una similitud entre los patrones de comportamiento involucrados en el consumo de un EI y los comportamientos dirigidos hacia el EC. En estudios clásicos con perros, se observó que el animal podía lamer la lámpara eléctrica (i.e., EC), o realizar tomas de aire por la boca como si quisiera consumir el sonido (Pavlov, 1932). En ratas se observó que cuando se presenta una palanca seguida de alimento, algunos animales se aproximan, la agarran y la roen como si fuera comida (Davey \& Cleland, 1982). En palomas se observó que al exponerse a una luz (i.e., EC) que se ha emparejado con la presentación de agua o alimento (i.e., EI) exhiben un patrón motor específico implicado en la conducta de beber o comer direccionado hacia la luz (Jenkins \& Moore, 1973). También se observó en machos de codorniz japonesa que al presentar un EC que anticipa la oportunidad de copular con una hembra (EI) se despliega la conducta de cópula con el objeto inanimado (i.e., EC; Burns \& Domjan, 1996, 2001).

Una de las primeras explicaciones sobre el automoldeamiento refería que el EC actúa como un sustituto del EI (Pavlov, 1927), dado que la presentación del EC a menudo conduce a una RC que se asemeja a un comportamiento causado por el EI mismo. Según esta idea, la capacidad elicitadora del EI se transfiere al EC, generando una reacción refleja equiparable (Jenkins \& Moore, 1973). Esto es consistente con la teoría que indica que el EC evoca estados emocionales $y$ motivacionales complejos y equivalentes al EI (Rescorla, 1988). Konorski (1948) propuso una distinción entre condicionamiento preparatorio y consumatorio. La RC preparatoria implica la expresión de conductas difusas que aluden al valor afectivo general del estímulo apetitivo o aversivo, y se despliegan con la presentación del EC asociado previamente al EI. En presencia de un EI apetitivo se genera un aumento en la activación y el despliegue de conductas de acercamiento. En cambio, un EI aversivo propicia la supresión de la conducta. Por su parte, las RC consumatorias dependen de las propiedades sensoriales del estímulo y se observan en conductas discreta y específicas que se despliegan frente a la presencia del EI apetitivo (e.g., deglutir, lamer o picotear) o aversivo (e.g., parpadear, escapar o saltar).

Una interpretación parsimoniosa del fenómeno de automoldeamiento señala que la clave, en lugar de propiciar un estado motivacional, simplemente conduciría a una respuesta refleja al igual que sucede con otras RC como la salivación o el parpadeo. Varios estudios muestran que patrones simples de movimiento responden a mecanismos del condicionamiento clásico. Por ejemplo, en el condicionamiento del reflejo palpebral los movimientos de parpadeo (i.e., RI) propiciados por un soplo de aire (i.e., EI) se 
asocian a un tono (i.e., EC), de modo tal que posteriores presentaciones del tono despliegan el reflejo (i.e., RC; e.g., Solomon, Pomerleau, Bennett, James, \& Morse, 1989; Schmaltz \& Theios, 1972; Solomon \& Moore, 1975). El patrón motor específico que se despliega en el automoldeamiento, equivalente a la conducta de beber o comer, podría ser una respuesta similar a la que ocurre en el reflejo palpebral incitado por el EC.

Sin embargo, otros estudios muestran que el automoldeamiento pareciera ser un fenómeno consistente con la idea según la cual el EC adquiere propiedades de incentivo provenientes del EI. Por ejemplo, en el paradigma de transferencia pavloviano a instrumental, los sujetos se exponen a ambos tipos de aprendizaje en contextos diferenciados. Se realiza un condicionamiento clásico en una situación diferente del condicionamiento instrumental para posteriormente evaluar el efecto del EC sobre el desempeño de la respuesta operante. El paradigma de transferencia consta de tres fases: entrenamiento pavloviano, entrenamiento instrumental y prueba de transferencia. En la fase pavloviana, uno o más EC se emparejan con la entrega del EI. Durante la fase instrumental, se establece una relación de contingencia entre una conducta y el reforzador. En la fase de transferencia, el animal puede realizar nuevamente las acciones instrumentales, en presencia de los EC (e.g., Campese, McCue, LázaroMuñoz, LeDoux, \& Cain, 2013; Colagiuri, \& Lovibond, 2015; Holmes, Marchand, \& Coutureau, 2010; Lewis, Niznikiewicz, Delamater, \& Delgado, 2013). Por ejemplo, Lovibond (1983) entrenó a conejos en dos tareas distintas, una instrumental y otra pavloviana. La fase de entrenamiento se dividió en dos momentos diferentes. Por un lado, los sujetos debían presionar una palanca para recibir una solución de sacarosa. Por el otro, seguido de la presentación de un sonido, se le daba acceso libre a la misma solución en ausencia de la palanca. Durante la fase de prueba se los expuso nuevamente a la tarea instrumental presentando ocasionalmente el sonido. Se observó que los conejos vigorizaban la respuesta de presionar la palanca cuando se les presentaba la clave. Lo interesante de este estudio es que la señal había potenciado la respuesta instrumental, en ausencia de una asociación pavloviana entre la respuesta motora (RC) y el tono (EC). En síntesis, el EC adquirió propiedades motivacionales de incentivo aumentando la respuesta necesaria para la obtención del estímulo incondicionado.

\section{Motivación de incentivo}

La teoría de Hull (1943) contempló dos formas diferenciadas de drive: una forma incondicionada y otra condicionada o adquirida. El drive incondicionado proviene del desbalance homeostático en relación a necesidades fisiológicas básicas, por lo que el organismo no precisa un aprendizaje previo para verse motivado por este impulso ya que el mismo constituye un mecanismo esencial para su supervivencia. Hull propuso que, a través del condicionamiento clásico, estímulos ambientales hasta el momento neutros pueden adquirir la habilidad de incentivos al producir estados internos de drive incitando la conducta. Este impulso es lo que se denomina drive condicionado. Por 
ejemplo, si un sujeto hambriento consigue su alimento en un determinado contexto, las claves contextuales y los estímulos ambientales característicos de este lugar pueden adquirir la capacidad de incitar motivación proveniente del drive condicionado. De esta manera, la presentación de estas claves podrá incitar la ingesta incluso en estado de saciedad.

Los incentivos no sólo son predictores del reforzador, sino que también funcionan como potenciadores de la ingesta. En la ingesta potenciada por clave, un estímulo discreto (EC) o claves contextuales asociados a un alimento (EI) en un estado de privación calórica puede potenciar la ingesta aun cuando sea presentado en un momento en el que los animales estén saciados de alimento (e.g., Cole, Keefer, Anderson \& Petrovich, 2020; Petrovich, Ross, Gallagher \& Holland, 2007; Weingarten, 1983).

En síntesis, los animales poseen mecanismos de aprendizaje sobre los estímulos del ambiente y las consecuencias de su conducta, incluida su valoración afectiva. De esta manera, utilizan dicha información para la satisfacción de sus necesidades primarias (López \& Cantora, 2005). La motivación de incentivo se basa en la anticipación de las propiedades afectivas de los estímulos incondicionados. Los estímulos ambientales pueden adquirir propiedades condicionadas de incentivo y generar estados motivacionales capaces de instigar la conducta. Además, estados fisiológicos extremos (e.g., hambre elevado) pueden aumentar el valor de incentivo de los estímulos meta, generando una interacción multiplicativa entre el déficit fisiológico y el estímulo externo (Toates, 1986, en Berridge, 2004). De este modo, el valor que posee un estímulo para el organismo y la motivación que el animal tendrá para su obtención queda determinado por múltiples fuentes motivacionales. El estado interno del animal (e.g., nivel de saciedad o hambre), la magnitud del estímulo (e.g., distintas concentraciones de solución azucarada) y las experiencias previas con este reforzador $\mathrm{u}$ otros de distinta magnitud y/o calidad influyen en la motivación del sujeto y, en consecuencia, en las conductas que despliegue para su obtención (Mackintosh, 1988).

\section{Otras fuentes de motivación: Necesidades psicológicas}

En el contexto de trabajos con humanos, algunos autores han incluido a las necesidades psicológicas como fuentes de motivación interna, al considerarlas como impulsos para alcanzar un objetivo deseado, tales como el logro, la filiación, la pertenencia a un grupo, el estatus o el poder (Deckers, 2001). Resulta discutible hasta qué punto pueden considerarse algunas de estas categorías como verdaderas fuentes internas de motivación, ya que algunas parecieran más bien ser categorías generales de incentivos y reforzadores. Por ejemplo, en el caso de la filiación, la experiencia de pertenencia y aceptación por un grupo puede contener un sinfín de reforzadores e incentivos sociales, también presentes en otras especies sociales. No obstante, muchas veces la conducta humana se encuentra impulsada por la consecución de metas abstractas que toman significado dentro de un contexto 
sociocognitivo y valorativo (e.g., motivación de logro, reconocimiento de pares). En estos casos, no son tan fácilmente identificables los eventos ambientales que gatillan la conducta, por lo que resulta razonable emparentar las necesidades psicológicas con las otras fuentes internas de motivación.

\section{Motivación extrínseca y motivación intrínseca: modelos animales}

Una segunda clasificación que surge en la literatura se centra en la dirección del comportamiento. Dependiendo del propósito, la conducta podría orientarse a una meta extrínseca o intrínseca. En términos operativos, la conducta extrínsecamente motivada implica una respuesta instrumental, se ejecuta para la obtención de un reforzador (e.g., Berridge, Robinson \& Aldridge, 2009; Castro \& Berridge, 2014a; Cuenya et al., 2015; Kamenetzky, Mustaca, \& Papini, 2008; Serafini \& Cuenya, 2017; Tantot et al., 2017) o la evitación de un estímulo aversivo (e.g., Holahan, 2004; Knapska et al, 2013; Stelly et al, 2019). En cambio, la conducta intrínsecamente motivada se observa cuando el comportamiento se realiza en ausencia de cualquier contingencia externa aparente (Morales Domínguez \& Gaviria Stewart, 1990). Sin embargo, que no se observen contingencias no implica en modo alguno que no existan, o que no existan de una forma encubierta. Algunos autores, como Aguado (2005), interpretan que cuando un escritor se sumerge, completamente absorto, en su actividad literaria por horas, logra un estado mental y emocional de satisfacción que puede entenderse como un reforzador encubierto y, como tal, estar sujeto parcialmente a las leyes generales del aprendizaje. De esta forma, la persistencia aparentemente independiente de la actividad se sostendría en el efecto reforzante del estado de satisfacción obtenido.

En la década de 1950 surgieron los primeros modelos animales que abordaron el estudio de la motivación intrínseca, en donde se estudiaban las conductas de exploración, clasificando los motivos como extrínsecos vs intrínsecos (Berlyne, 1960). La exploración motivada en forma extrínseca despliega un comportamiento dirigido hacia una meta externa en respuesta a algún requerimiento específico. Implica la búsqueda activa de un resultado particular, como encontrar comida en respuesta al hambre o encontrar un medio de escape de un ambiente peligroso. La exploración motivada de forma intrínseca refleja la tendencia a la búsqueda de la novedad, búsqueda de sensaciones, la curiosidad y el aprendizaje, en ausencia de reforzadores externos y relaciones aparente de contingencia con reforzadores o incentivos (Hughes, 1997).

La literatura sobre los fenómenos básicos del aprendizaje abordó la motivación intrínseca indagando los mecanismos implicados. En un inicio se asumió que estaba gobernada por mecanismos equivalentes al drive incondicionado. Uno de los primeros estudios en abordarlo la definió como drive de exploración. En una investigación clásica de Harlow (1950), se evaluó el aprendizaje en monos rhesus del armado de rompecabezas mecánicos complejos en ausencia de reforzadores externos. Los 
resultados evidenciaron no sólo una mejora progresiva en el rendimiento de la tarea motivada por una recompensa intrínseca (i.e., decremento del impulso de exploración), sino también un mantenimiento de la conducta por periodos de 10 horas en ausencia de reforzadores externos. Tales datos llevaron a la idea de que ciertos tipos de respuesta exploratoria pueden surgir de un impulso de exploración independiente de la satisfacción de necesidades fisiológicas específicas (Myers \& Miller, 1954).

Si la motivación intrínseca se presenta en ausencia de refuerzos externos y relaciones aparente de contingencia con reforzadores o incentivos ¿cuáles son los motivos que incitan esta conducta de exploración?, ¿cómo se sostiene?, ¿puede proponerse la existencia de una necesidad de estimulación sensorial? Algunos estudios sobre reforzamiento sensorial evidencian que el estímulo novedoso, en sí mismo, puede funcionar como un estímulo incondicionado o reforzador apetitivo que aumenta la probabilidad de ocurrencia de una respuesta instrumental. Por ejemplo, ratas, ratones y monos despliegan una conducta de palanqueo reforzada por la presentación de un estímulo novedoso (e.g., luz o sonido) (Barnes \& Kish, 1961; Kish, 1955; Marx, Henderson, \& Roberts, 1955; Moon \& Lodahl, 1956; Robinson, 1961). Por su parte, los monos rhesus aprenden a discriminar colores para tener la oportunidad de abrir una puerta y observar fuera de la cámara experimental a un grupo de personas (Butler, 1953), un tren de juguete en movimiento (Butler, 1954) o a una colonia de monos (Butler \& Alexander, 1955). En otros trabajos se indagó si la exploración es incitada por la estimulación novedosa o por mecanismos de privación y saciedad sensorial. Para esto se entrenaron ratas en el recorrido de un laberinto en forma de T. Durante la fase de exposición, un brazo era de color negro y el otro blanco. Se colocó un vidrio transparente que bloqueaba el acceso a los brazos. De esta forma las ratas podían observarlos, pero no ingresar a ellos. En la fase de elección se les permitió el acceso, pero uno de los brazos había cambiado, de forma tal que ambos eran negros o blancos. Si las ratas se hubieran saciado a los estímulos familiares, no deberían mostrar preferencia por alguno de los brazos. Sin embargo, exploraron más el brazo que cambió de color, a pesar de que en ese momento eran exactamente iguales, lo que sugiere que la exploración es incitada por la estimulación novedosa más que por mecanismos de privación sensorial (Dember, 1956).

La motivación intrínseca no responde a los mismos mecanismos que gobiernan al drive incondicionado. En este último, la privación fisiológica incita la conducta necesaria para obtener el estímulo que favorece el retorno al estado homeostático. En la motivación intrínseca sucede lo opuesto, la privación fisiológica no es la que incita la conducta de exploración sino el estímulo novedoso (Brown, 1961). Si los estímulos externos son los que propician el impulso de exploración, la conducta podría estar gobernada por mecanismos equivalentes al drive condicionado. No obstante, el drive de exploración surge en forma espontánea ante estímulos novedosos. El drive de exploración difiere en varios aspectos del drive homeostático (e.g., el hambre y la sed). En primer lugar, 
tiene un arousal exteroceptivo, lo que implica que la exploración es incitada por el ambiente externo del animal. Otra diferencia yace en las consecuencias de la conducta motivada. El drive homeostático (e.g., sed) se reduce con la estimulación (e.g., agua), mientras que el drive exploratorio incrementa a medida que aparecen estímulos novedosos (Fowler, 1965).

En suma, se observa que este tipo de conducta es impulsada por la novedad que se genera ante cambios espaciales y temporales más que por una necesidad homeostática o autoregulatoria de estimulación. En cuanto a la dirección del comportamiento, la respuesta está orientada a la realización de la tarea (i.e., explorar) en ausencia de cualquier contingencia externa aparente, por lo que puede ser comprendida como parte de la motivación intrínseca.

\section{Motivación extrínseca e intrínseca en humanos}

El comportamiento humano, merced al desarrollo de la conducta verbal, tiene niveles de complejidad superior al del resto de los animales, por lo que resulta importante repasar los modelos psicológicos que abordan la diferencia entre motivación extrínseca e intrínseca en el comportamiento humano. Esta distinción resulta un componente crucial en las teorías contemporáneas, principalmente en la tradición sociocognitiva y aplicada.

Uno de los autores pioneros más influyentes en relación a la motivación intrínseca humana es Edward Deci. El autor refiere que ésta requiere dos elementos centrales: la competencia y la autodeterminación, ambas consideradas necesidades psicológicas de los individuos. La autodeterminación tiene un rol preponderante, para que una actividad resulte intrínsecamente motivante no es suficiente sentir en ella un buen nivel de competencia, sino también se debe percibir autonomía en su ejecución, en otras palabras, un locus interno de causalidad percibida (Deci, 1975). Años más tarde, Ryan y Deci (2000) propusieron un modelo taxonómico de la motivación humana. El modelo plantea un continuo entre la amotivación y la motivación intrínseca, atravesando distintas fuentes motivacionales que habitualmente se colocan en la motivación extrínseca.

Según este modelo, la amotivación constituye la ausencia total de interés e intención de realizar una conducta, ausencia de valoración por la misma, de sentimiento de competencia para ejecutarla y expectativas de consecuencias deseadas. Un nivel superior en términos motivacionales lo constituye la regulación externa, en la que la conducta se ejecuta exclusivamente para la obtención de una consecuencia deseada. Es el tipo de motivación que en los estudios clásicos es considerada como motivación extrínseca y sobre la que versa la mayoría de los trabajos en psicología experimental y neurociencia conductual, en los que se evalúa la respuesta ante la presentación de incentivos y/o reforzadores. Lo interesante del modelo es que incorpora entre la regulación externa y la motivación intrínseca otras categorías intermedias. En un nivel superior, los autores ubican a la 
regulación introyectada. Este tipo de motivación es la que mueve a un sujeto a realizar una tarea para evitar la culpa o ansiedad de no realizarla, o para conservar o ganar cierto grado de autoestima o valor. Una forma de mayor autonomía de la motivación extrínseca la constituye la identificación, en la cual el individuo incorpora la importancia de la conducta y la ejecuta por su propia decisión ya que la considera valiosa para la consecución de alguna meta, aunque la actividad no le resulte inherentemente placentera. Finalmente, la regulación integrada constituye la forma más autónoma de motivación extrínseca, una identificación completa del sujeto con la actividad, ejecutada por una decisión autónoma en función de su congruencia con otras necesidades y objetivos del individuo. Sin embargo, la conducta continúa siendo ejercida por su función instrumental, en el corto o largo plazo. La motivación intrínseca sería el último escalón en este modelo, el que se desplaza de un mínimo a un máximo de autodeterminación y locus de causalidad interno (ver Ryan \& Deci, 2000).

Resulta importante realizar algunas consideraciones sobre las implicancias modelo. En primer lugar, se aparta de la lógica binaria de estudios previos (motivación extrínseca $v s$ intrínseca) y de conceptos unitarios sobre la motivación, puesto que propone una dimensión continua desde la amotivación hasta la motivación intrínseca. En segundo lugar, y tal como expresan los autores, el modelo no debe ser entendido como un desarrollo de las motivaciones en cualquier tipo de tarea, sino como un gradiente de autodeterminación y locus de causalidad percibida. No es esperable que todas las actividades $\mathrm{o}$ conductas puedan ser impulsadas y/o sostenidas por motivación intrínseca, ni que todas puedan ganar en cuanto a su nivel de autonomía, aunque sí existen situaciones en las que esto ocurra.

\section{Subsistemas motivacionales y sus bases neurobiológicas}

\section{Liking y Wanting}

Una distinción importante que surge en la literatura neuroconductual diferencia dos grandes subsistemas motivacionales relacionados con las fuentes de motivación externa. Los reforzadores alimenticios tienen dos componentes que están asociados con la palatabilidad (i.e, el placer sensorial al comer) y el apetito (i.e., disposición a comer; Rogers, 1990). Estos son definidos habitualmente en los estudios sobre neurociencia conductual como liking $\mathrm{y}$ wanting, respectivamente. El liking es esencialmente el impacto hedónico o afectivo, la reacción cerebral subyacente al placer sensorial producto de la presencia de un estímulo apetitivo, como, por ejemplo, un sabor dulce. El wanting se define como la saliencia de incentivo motivacional $y$ promueve la predicción de situaciones placenteras, orientando al organismo para ejecutar las conductas pertinentes para obtener el refuerzo (Berridge, 1996; Berridge, 2009a; Berridge, 2009b; Castro \& Berridge, 2014a).

El liking $\mathrm{y}$ wanting son dos componentes que suelen coexistir en una misma situación de aprendizaje. La mayoría 
de los trabajos que utilizan el consumo de un refuerzo alimenticio como medida dependiente no permite evaluar diferencialmente estos subsistemas motivacionales. Evitar el consumo de un alimento o consumirlo en forma exacerbada puede implicar un cambio de su valor hedónico, un cambio en su saliencia de incentivo o cambios en ambos componentes. Las medidas tradicionales del valor motivacional del refuerzo alimenticio van desde la ingesta del reforzador hasta el registro de las acciones necesarias que el animal deberá realizar para obtenerlo, pero ¿se capta el mismo fenómeno al registrar la ingesta de un refuerzo que al medir la conducta de palanqueo para obtenerlo? Realizar este tipo de distinciones es relevante para comprender de forma específica la regulación del apetito y dar una explicación detallada de los aspectos involucrados, por ejemplo, en algunos desórdenes de la conducta alimentaria y el mantenimiento de patrones de ingesta disfuncionales. La psicología experimental ha aportado protocolos específicos que permiten estudiar de forma desagregada estos dos subsistemas. A su vez, el estudio de sus sustratos neurobiológicos muestra que son fenómenos diferenciados asociados a mecanismos neuronales específicos. Por ejemplo, la interrupción de los sistemas dopaminergicos mesolímbicos reduce la saliencia de incentivo o wanting sin afectar al impacto hedónico o liking (Berridge \& Robinson, 1998; Peciña, Berridge \& Parker, 1997).

Un buen indicador del wanting o saliencia de incentivo es el breakpoint. Este es el punto en el que los animales dejan de responder a la recompensa en un programa de razón. En otras palabras, es el esfuerzo que el animal está dispuesto a realizar para conseguir un refuerzo (e.g., Clark \& Bernstein, 2006; Lorrain, Arnold \& Vezina, 2000).

El liking se puede abordar, en ratas, por medio del estudio de la microestructura de la conducta consumatoria midiendo la cantidad de lameteos por burst (ráfaga de lameteos), el cual es un buen indicador del valor hedónico (Grigson, Spector, \& Norgren, 1993). Otro indicador objetivo para estudiar el liking es la conducta orofacial que se despliega ante la degustación de estímulos palatables o desagradables. Estas reacciones faciales parecieran haberse desarrollado a partir de la misma fuente evolutiva en humanos, orangutanes, chimpancés, monos, ratas y ratones (Berridge, 2000; Steiner, Glaser, Hawilo, \& Berridge, 2001). La conducta orofacial tiene patrones de respuesta específicos que se pueden clasificar en patrones asociados a estímulos apetitivos o aversivos. El repertorio conductual específico de respuestas que conforma cada patrón varía en función de capacidad muscular, sensorial y los hábitos alimenticios de la especie que se esté estudiando. Por ejemplo, en ratas se pueden inferir los patrones asociados a estímulos apetitivos por la observación de protrusión de lengua, movimiento de boca y lameteo de las patas delanteras. Los patrones aversivos aluden a comportamientos como el goteo pasivo, arcadas, frotamiento de barbilla, movimiento de cabeza, movimiento de patas delanteras, movimientos de extremidades anteriores o limpieza con las patas (e.g., Cuenya, Bura, Serafini, \& López, 
2018; Grill \& Norgren, 1978; Parker, 1994; Pfaffmann \& Norgren, 1977; Suárez, Mustaca, Pautassi, \& Kamenetzky, 2014).

\section{Circuitos neurobiológicos implicados}

Como se anticipó en el apartado anterior, el liking y wanting tienen circuitos neurobiológicos especializados. Los sustratos y mecanismos neurobiológicos que conciernen al wanting se relacionan con vías dopaminérgicas (Wyvell \& Berridge, 2001) y se encuentran ampliamente distribuidos por el encéfalo $y$, en ocasiones, se superponen con aquellos implicados en el liking (e.g., en el sistema límbico; ver Castro \& Berridge, 2014a; Berridge et al., 2009). Por ejemplo, en un estudio llevado a cabo por Wyvell y Berridge (2000) se entrenó a las ratas para presionar una de dos palancas para obtener pellets de sacarosa. En la fase de prueba se les aplicó una microinyección bilateral de anfetamina al interior del NAc y se registró un aumento de la conducta de palanqueo en ausencia de la contingencia del refuerzo. Estos resultados evidencian que en el NAc la dopamina interviene específicamente en la capacidad de las señales para desencadenar el wanting de la recompensa, independientemente del impacto hedónico asociado a patrones apetitivos.

En lo que respecta al liking son preponderantes los sistemas opioides (e.g., Peciña \& Berridge, 2005; Richardson, Reynolds, Cooper, \& Berridge, 2005), endocanabinoides (Mahler, Smith \& Berridge, 2007) y GABAergicos (e.g., Cooper \& Ridley, 2005; Ho \& Berridge, 2014; Reynolds \& Berridge, 2002; Smith \& Berridge, 2005). Por ejemplo, las microinyecciones de un agonista opioide (Peciña \& Berridge, 2005) o GABAérgico (Reynolds \& Berridge, 2002) en la zona rostrodorsal del área media de la corteza del NAc amplifica los patrones apetitivos hacia los sabores dulces. Asimismo, se amplifica el efecto con inyecciones intraperitoneales de un agonista de receptores benzodiacepinicos (Cooper \& Ridley, 2005). Las inyecciones intraperitoneales de diazepam (i.e., agonista de GABA) aumenta los patrones apetitivos $\mathrm{y}$ disminuye los patrones aversivos hacia una solución de sacarosaquinina, en comparación con ratas que recibieron el vehículo. Además, la aplicación de naltrexona, antagonista opioide, revierte el efecto inducido por el diazepam. Estos resultados sugieren que ambos sistemas interactúan en la expresión de las respuestas de reacción al sabor (Richardson et al., 2005).

\section{Bases Neuroanatómicas}

Las estructuras cerebrales vinculadas al liking se restringen principalmente al área rostromedial de la corteza del NAc y la zona caudal del pálido ventral (Cromwell \& Berridge, 1993; Peciña \& Berridge, 2005; Tindell, Smith, Peciña, Berridge, \& Aldridge, 2006). Entre estas estructuras se localizan áreas anatómicas especializadas en la configuración y la modulación del valor hedónico del refuerzo. Estas áreas se denominan puntos hedónicos (ver Figura 1) y su estimulación farmacológica puede causar una modulación de las reacciones asociadas al liking. Por ejemplo, la inactivación temporal mediante la aplicación de un antagonista de GABA en la mitad caudal de la zona medial de la corteza 
del NAc potencia los patrones aversivos hacia la sacarosa (Ho \& Berridge, 2014; Reynolds \& Berridge, 2002). Por otro lado, microinyecciones de agonistas opioides potencian los patrones apetitivos (Castro \& Berridge, 2014b).

El pálido ventral tiene un rol central en los circuitos de placer $y$ en la configuración del valor hedónico de la recompensa. Por ejemplo, la microinyección de opioides u orexinas en esta zona puede duplicar los patrones hedónicos asociados a respuestas apetitivas ante la degustación de sabores dulces (Ho \& Berridge, 2013; Smith \& Berridge, 2005, 2007). La inactivación temporal de GABA en la mitad posterior genera fuertes respuestas aversivas ante la sacarosa (Ho \& Berridge, 2014). Otra área cerebral relevante en la modulación del liking es el núcleo pontino parabraquial, cuya estimulación con benzodiacepinas potencia la ingesta $\mathrm{y}$ las conductas hedónicas asociadas a patrones apetitivos frente a la sacarosa (Higgs \& Cooper 1996; Peciña \& Berridge 1996; Söderpalm \& Berridge, 2000).

Algunos estudios evaluaron si lesiones de áreas implicadas en el sistema gustativo, como la amígdala, están asociadas al liking. Se halló que las lesiones bilaterales del núcleo central de la amígdala (Gavalerna et al., 1993; Rana \& Parker, 2008) y de la amígdala basolateral (Rana \& Parker, 2008) no afectaron las reacciones apetitivas generadas por el impacto hedónico ante la degustación de los estímulos (salado, dulce, acido, amargo). Tampoco las lesiones de zonas próximas como el hipotálamo lateral afectaron al liking, pero las lesiones en el globo pálido ventral produjeron un aumento en los patrones aversivos hacia la sacarosa (Cromwell \& Berridge, 1993). Sin embargo, microinyecciones de un agonista opioide en la mitad posterior del pálido ventral generan un aumento de patrones apetitivos hacia la sacarosa, mientras que microinyecciones en zonas anterior $y$ central los suprimen (Smith \& Berridge, 2005). Otros estudios muestran que tanto lesiones como inactivaciones farmacológicas realizadas en la mitad posterior del pálido ventral propician un aumento desmedido de patrones aversivos hacia la sacarosa (Ho \& Berridge, 2014).

\section{Figura 1}

Puntos hedónicos

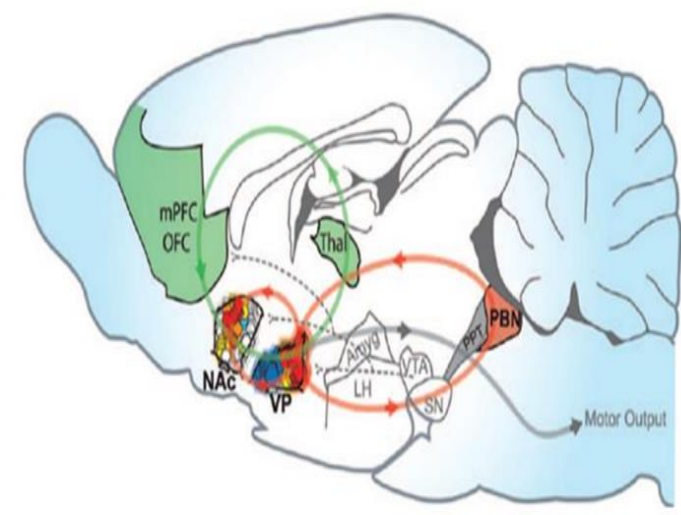

Nota. Las proyecciones de los circuitos hedónicos se presentan mediante líneas que conectan los distintos puntos hedónicos (i.e., círculos rojos) o generando un bucle más grande dentro del sistema mesocorticolímbico (i.e., flechas verdes). NAc = Corteza del núcleo accumbens; VP = Pálido ventral; $\mathrm{mPFC}=$ Corteza pre-frontal medial; $\mathrm{OFC}=$ Corteza orbitofrontal; Thal = tálamo; LH = Hipotálamo lateral; Amyg = Amígdala; $\mathrm{SN}=$ sustancia nigra; VTA = Área tegmental ventral; PPT = Tegmento pedunculopontino; $\mathrm{PBN}=$ núcleo parabraquial (Peciña, Smith, \& Berridge, 2006) 
Las

$$
\text { estructuras }
$$

cerebrales

vinculadas al wanting incluyen principalmente a los circuitos dopaminergicos del NAc y pálido ventral. El bloqueo de dopamina con pimozida (Peciña et al., 1997) o lesiones neurotóxicas con 6OHDA (Berridge \& Robinson, 1998; Berridge, Venier, \& Robinson, 1989) en el NAc producen una merma del wanting sin afectar el liking. Por otro lado, microyencciones de anfetamina en la corteza del NAc (Smith, Berridge \& Aldridge, 2011; Wyvell \& Berridge, 2000) y el globo pálido ventral lo aumentan (Tindell et al., 2006).

\section{Conclusión}

Esta revisión abordó los aportes realizados por las principales teorías psicológicas en el área de la motivación con el objetivo de clarificar el mapa conceptual desde el que se desprenden los conceptos motivacionales utilizados en la actualidad. Una de las razones por las cuales resulta difícil abarcar el concepto en una única definición consensuada reside en que no es un constructo unitario, sino más bien un conjunto de mecanismos y subsistemas que impulsan y dirigen la conducta. Si bien dentro del estudio de la motivación hay divisiones que pueden aparentar artificiales, el desarrollo de protocolos experimentales y de medidas apropiadas posibilita su abordaje en forma diferenciada. En este sentido, los modelos animales han colaborado en clarificar los mecanismos intervinientes.

La motivación no debe ser comprendida como una serie de categorías aisladas o contrapuestas, sino como un sistema multicomponente que energiza y direcciona la conducta a la consecución de una meta. El comportamiento puede orientarse a obtener o evitar un estímulo ambiental (i.e., motivación extrínseca) o las sensaciones que provoca la misma actividad (i.e., motivación intrínseca). De esta forma, se puede entender a la motivación como una dimensión continua cuyos extremos son la amotivación y la motivación intrínseca, atravesando distintas fuentes motivacionales que habitualmente se colocan en la motivación extrínseca. La fuente que impulsa motivacionalmente al organismo puede ser interna, por ejemplo, en el caso de las conductas traccionadas por regulación homeostática o drive incondicionado (e.g., alimentarse ante el déficit energético), o puede ser una fuente externa, ya sea por la presentación de un estímulo incondicionado intenso, o estímulos condicionados (drive condicionado o motivación de incentivo). A su vez, en el contexto de los reforzadores alimenticios, las fuentes motivacionales externas se asocian a bases neurobiológicas diferenciadas. Por un lado, el componente hedónico del refuerzo - liking - se asocia a circuitos opioides, endocanabinoides $\mathrm{y}$ gabaergicos. Por el otro, la saliencia de incentivo motivacional - wanting - se asocia a vías dopaminérgicas.

Los mecanismos fundamentales que gobiernan y energizan la conducta son filogenéticos primitivos y, en consecuencia, se encuentran presentes en un amplio rango de especies. Dada la inscripción sociocultural y lingüística de buena parte de los reforzadores e incentivos humanos, la complejidad de las motivaciones humanas nunca podrá ser capturada cabalmente por 
un modelo animal. Sin embargo, estos han constituido una herramienta experimental valiosa para dilucidar mecanismos motivacionales generales. Por su parte, la neurociencia conductual ha permitido en las últimas décadas clarificar los sustratos materiales de buena parte de estos mecanismos. Intentar encontrar elementos en común entre marcos teóricos diferentes

\section{References}

Aguado, L. (2005). Emoción, afecto y motivación. Alianza Editorial.

Autio, 0. (2011). Elements in students motivation in technology education. Procedia - Social and Behavioral Sciences, 29, 1161-1168. https://doi.org/10.1016/j.sbspro.2 011.11.350.

Barnes, G. W., \& Kish, G. B. (1961). Reinforcing properties of the onset of auditory stimulation. Journal of Experimental Psychology, 62(2), 164-

170. https://doi.org/10.1037/h00 42091.

Beck, R. C. (2000). Motivation. theories \& principles (4ta ed.). Prentice Hall.

Berlyne, D. E. (1960). Conflict, arousal, and curiosity. McGraw-Hill. https://doi.org/10.1037/11164$\underline{000}$.

Berridge, K. C. (2000). Measuring hedonic impact in animals and infants: microstructure of affective taste reactivity patterns. Neuroscience \& y criterios demarcatorios de conceptos motivacionales facilita una tarea de por sí ardua: la vinculación de la investigación básica en contextos controlados de laboratorio y la investigación aplicada.

Biobehavioral Reviews, 24(2), 173198. https://doi.org/10.1016/s014 9-7634(99)00072-x.

Berridge, K. C. (1996). Food reward: Brain substrates of wanting and liking. Neuroscience \& Biobehavioral Reviews, 20(1), 125. https://doi.org/10.1016/01497634(95)00033-b

Berridge, K. C. (2004). Motivation concepts in behavioral neuroscience. Physiology \& Behavior, 81(2), 179209.

https://doi.org/10.1016/j.physbeh .2004 .02 .004 .

Berridge, K. C. (2009a). 'Liking' and 'wanting' food rewards: Brain substrates and roles in eating disorders. Physiology \& Behavior, 97(5), 537-550. https://doi.org/10.1016/j.physbeh .2009 .02 .044 .

Berridge, K. C. (2009b). Wanting and liking: Observations from the neuroscience and psychology laboratory. Inquiry, 52(4), 378-398. 
https://doi.org/10.1080/0020174 0903087359.

Berridge, K. C., \& Robinson, T. E. (1998). What is the role of dopamine in reward: hedonic impact, reward learning, or incentive salience? Brain Research Reviews, 28(3), 309369.

https://doi.org/10.1016/s01650173(98)00019-8.

Berridge, K. C., Venier, I. L., \& Robinson, T. E. (1989). Taste reactivity analysis of 6-hydroxydopamine-induced aphagia: Implications for arousal and anhedonia hypotheses of dopamine function. Behavioral Neuroscience, 103(1), 3645. https://doi.org/10.1037/0735$\underline{7044.103 .1 .36}$.

Berridge, K.C., Robinson, T.E., \& Aldridge, J.W. (2009). Dissecting components of reward: 'liking', 'wanting', and learning. Current Opinion in Pharmacology, 9, 65-73. https://doi.org/10.1016/j.coph.20 $\underline{08.12 .014}$.

Brown, J. S. (1961). The motivation of behavior. McGraw-Hill.

Burns, M., \& Domjan, M. (1996). Sign tracking versus goal tracking in the sexual conditioning of male Japanese quail (Coturnix japonica). Journal of Experimental Psychology: Animal Behavior Processes, 22(3), 297-306. https://doi.org/10.1037/00977403.22.3.297.
Burns, M., \& Domjan, M. (2001). Topography of spatially directed conditioned responding: Effects of context and trial duration. Journal of Experimental Psychology: Animal Behavior Processes, 27(3), 269-

278. https://doi.org/10.1037/009 7-7403.27.3.269.

Butler, R. A. (1953). Discrimination learning by rhesus monkeys to visualexploration motivation. Journal of Comparative and Physiological Psychology, 46(2), 9598. https://doi.org/10.1037/h006 1616.

Butler, R. A. (1954). Incentive conditions which influence visual exploration. Journal of Experimental Psychology, 48(1),

19

23. https://doi.org/10.1037/h006 $\underline{3578 .}$.

Butler, R. A., \& Alexander, H. M. (1955). Daily patterns of visual exploratory behavior in the monkey. Journal of Comparative and Physiological Psychology, 48(4), 247-

249. https://doi.org/10.1037/h00 $\underline{45210}$.

Campese, V., McCue, M., Lázaro-Muñoz, G., LeDoux, J. E., \& Cain, C. K. (2013). Development of an aversive Pavlovian-to-instrumental transfer task in rat. Frontiers in Behavioral Neuroscience, $\quad 7, \quad 1-10$. https://doi.org/10.3389/fnbeh.20 13.00176. 
Castro, D. C., \& Berridge, K. C. (2014b). Opioid hedonic hotspot in nucleus accumbens shell: Mu, Delta, and Kappa maps for enhancement of sweetness "liking" and "wanting". Journal of Neuroscience, 34(12), 4239-4250.

https://doi.org/10.1523/ineurosci. 4458-13.2014.

Castro, D.C., \& Berridge, K.C. (2014a). Advances in the neurobiological bases for food 'liking' versus 'wanting'. Physiology \& Behavior, 136, 22-30

https://doi.org/10.1016/j.physbeh .2014 .05 .022 .

Clark, J. J., \& Bernstein, I. L. (2006). Sensitization of salt appetite is associated with increased "wanting" but not "liking" of a salt reward in the sodium-deplete rat. Behavioral Neuroscience, 120(1), 206210. https://doi.org/10.1037/073 5-7044.120.1.206.

Colagiuri, B., \& Lovibond, P. F. (2015). How food cues can enhance and inhibit motivation to obtain and consume food. Appetite, 84, 7987. https://doi.org/10.1016/j.appe t.2014.09.023.

Cole, S., Keefer, S. E., Anderson, L. C., \& Petrovich, G. D. (2020). Medial prefrontal cortex neural plasticity, orexin receptor 1 signaling, and connectivity with the lateral hypothalamus are necessary in cuepotentiated feeding. The Journal of Neuroscience, 40(8), 1744-
1755. https://doi.org/10.1523/ine urosci.1803-19.2020.

Collins, R., Lanham, R. A., \& Sigford, B. J. (2000). Reliability and validity of the Wisconsin HSS Quality of Life Inventory in traumatic brain injury. Journal of Head Trauma Rehabilitation, 15(5), 11391148. https://doi.org/10.1097/00 001199-200010000-00007.

Cooper, S. J. (2008). From Claude Bernard to Walter Cannon. Emergence of the concept of homeostasis. Appetite, 51(3),

419-

427. https://doi.org/10.1016/j.app et.2008.06.005.

Cooper, S. J., \& Ridley, E.T. (2005). Abecarnil and palatability: Taste reactivity in normal ingestion in male rats. Pharmacology, Biochemistry and Behavior, 81, 517 - 523. https://doi.org/10.1016/j.pbb.200 5.02.014.

Cromwell, H. C., \& Berridge, K. C. (1993) Where does damage lead to enhanced food aversion: the ventral pallidum/substantia innominata or lateral hypothalamus?. Brain Research, 624, 1-10. https://doi.org/10.1016/00068993(93)90053-P.

Cuenya, L., Bura, S., Serafini, M., \& López, M. (2018). Consummatory successive negative contrast in rats: assessment through orofacial taste reactivity responses. Learning and Motivation, 63, 98-104. 
https://doi.org/10.1016/j.lmot.20 18.04.001.

Cuenya, L., Sabariego, M., Donaire, R., Fernandez-Teruel, A., Torres, C., \& Papini, M. R. (2015). Transfer across reward devaluation tasks in inbred roman rat strains. Learning and Motivation, 52, 22-31. https://doi.org/10.1016/j.lmot.20 $\underline{15.08 .003}$

Davey, G. C., \& Cleland, G. G. (1982). Topography of signal-centered behavior in the rat: effects of deprivation state and reinforcer type. Journal of the experimental analysis of behavior, 38(3), 291-304. https://doi.org/10.1901/jeab.1982 .38-291.

Deci, E. L. (1975). Intrinsic motivation. Plenum Press.

Deckers, L. (2001). Motivation. biological, psychological, and environmental. Allyn \& Bacon.

Dember, W. N. (1956). Response by the rat to environmental change. Journal of comparative and physiological psychology, 49(1), 93-95. https://doi.org/10.1037/h004541 1.

Di Ciano, P., \& Everitt, B. J. (2004). Conditioned reinforcing properties of stimuli paired with selfadministered cocaine, heroin or sucrose: implications for the persistence of addictive behaviour. Neuropharmacology, 47, 202-213. https://doi.org/10.1016/i.neuroph arm.2004.06.005.

Dickinson, A., Smith, J., \& Mirenowicz, J. (2000). Dissociation of Pavlovian and instrumental incentive learning under dopamine antagonists. Behavioral Neuroscience, 114(3), 468-483. https://doi.org/10.1037/07357044.114.3.468.

Domjan, M. (2010). Condicionamiento clásico: Fundamentos. En M. E., Ortiz Salinas (Ed.), Principios de aprendizaje y conducta (pp. 67103). Cengage Learning.

Flagel, S. B., \& Robinson, T. E. (2017). Neurobiological basis of individual variation in stimulusreward learning. Current Opinion in Behavioral Sciences, 13, 178185. https://doi.org/10.1016/i.cob eha.2016.12.004.

Flagel, S. B., Akil, H., \& Robinson, T. E. (2009). Individual differences in the attribution of incentive salience to reward-related cues: Implications for

addiction. Neuropharmacology, 56, 139-148.

https://doi.org/10.1016/j.neuroph arm.2008.06.027.

Flagel, S. B., Watson, S. J., Akil, H., \& Robinson, T. E. (2008). Individual differences in the attribution of incentive salience to a rewardrelated cue: Influence on cocaine sensitization. Behavioural Brain Research, 186(1), 48-56. 
https://doi.org/10.1016/j.bbr.200 7.07.022.

Fowler, H., (1965). Curiosity and Exploratory Behavior. Macmillan.

Freud, S. (1915). Pulsiones y destinos de pulsión. En J. L. Etcheverry (1976), Contribución a la Historia del Movimiento Psicoanalítico. Obras Completas. Tomo XIV. Amorrortu Editores.

Gavalerna, O. G., Seeley, R. J., Berridge, K. C., Grill, H. J., Epstein, A. N., \& Schulkin, J. (1993). Lesions of the central nucleus of the amygdala I: effects on taste reactivity, taste aversion learning and sodium appetite. Behavioural Brain Research, 59, 1117. https://doi.org/10.1016/01664328(93)90146-H.

Grigson, P. S., Spector, A. C., \& Norgren, R. (1993). Microstructural analysis of successive negative contrast in freefeeding and deprived rats. Physiology \& Behavior, 54(5), 909916.

\section{https://doi.org/10.1016/0031- 9384(93)90301-U.}

Grill, H. J., \& Norgren, R. (1978). The taste reactivity test. I. Mimetic responses to gustatory stimuli in neurologically normal rats. Brain Research, 143(2), 263-279. https://doi.org/10.1016/00068993(78)90568-1.

Guarda, A. S., Schreyer, C. C., Boersma, G. J., Tamashiro, K. L., \& Moran, T. H. (2015). Anorexia nervosa as a motivated behavior: Relevance of anxiety, stress, fear and learning. Physiology \& Behavior, 152, 466472. https://doi.org/10.1016/j.phy sbeh.2015.04.007.

Harlow, H. F. (1950). Learning and satiation of response in intrinsically motivated complex puzzle performance by monkeys. Journal of Comparative and Physiological Psychology, 43(4), 289294. https://doi.org/10.1037/h00 58114.

Higgs, S., \& Cooper, S. J. (1996). Hyperphagia induced by direct administration of midazolam into the parabrachial nucleus of the rat. European journal of pharmacology, 313(1-2), 1-9. https://doi.org/10.1016/00142999(96)00446-3.

Ho, C. Y., \& Berridge, K. C. (2013). An orexin hot spot in ventral pallidum amplifies hedonic "liking" for sweetness.

Neuropsychopharmacology, 39, 1655-1664.

Ho, C. Y., \& Berridge, K. (2014). Excessive disgust caused by brain lesions or temporary inactivations: Mapping hotspots of nucleus accumbens and ventral pallidum. European Journal of Neuroscience, 40, 3556-3572. https://doi.org/10.1111/ejn.1272 0 .

Holahan, M. R. (2004). Intra-Amygdala muscimol injections impair freezing and place avoidance in aversive contextual conditioning. Learning \& 
Memory, 11(4), 436446. https://doi.org/10.1101/lm.6 4704.

Holmes, N. M., Marchand, A. R., \& Coutureau, E. (2010). Pavlovian to instrumental transfer: A neurobehavioural perspective. Neuroscience \& Biobehavioral Reviews, 34(8), 1277 1295. https://doi.org/10.1016/j.ne ubiorev.2010.03.007.

Holsen, L., Lawson, E., Blum, J., Ko, E., Makris, N., Fazeli, P., ... Goldstein, J. (2012). Food motivation circuitry hypoactivation related to hedonic and nonhedonic aspects of hunger and satiety in women with active anorexia nervosa and weightrestored women with anorexia nervosa. Journal of Psychiatry \& Neuroscience, 37(5), 322332. https://doi.org/10.1503/jpn.1 10156.

Hughes, R. N. (1997). Intrinsic exploration in animals: motives and measurement. Behavioural Processes, 41(3), 213-226. https://doi.org/10.1016/S03766357(97)00055-7.

Hull, C. L. (1943). Principles of behavior. Appleton.

Jenkins, H. M., \& Moore, B. R. (1973). The form of the auto-shaped response with food or water reinforcers. Journal of the Experimental Analysis of Behavior, 20(2), 163181. https://doi.org/10.1901/jeab. 1973.20-163.
Kamenetzky, G., Mustaca, A., \& Papini, M. (2008). An analysis of the anxiolytic effects of ethanol on consummatory successive negative contrast. Avances en Psicología Latinoamericana, 26(2), 135-144.

Kish, G. B. (1955). Learning when the onset of illumination is used as the reinforcing stimulus. Journal of Comparative and Physiological Psychology, 48(4), 261264. https://doi.org/10.1037/h00 40782.

Knapska, E., Lioudyno, V., Kiryk, A., Mikosz, M., Gorkiewicz, T., Michaluk, P., ... Kaczmarek, L. (2013). Reward learning requires activity of matrix metalloproteinase- 9 in the central amygdala. Journal of Neuroscience, $33(36)$, $14591-$ 14600. https://doi.org/10.1523/in eurosci.5239-12.2013.

Konorski, J. (1948). Conditioned reflexes and neuron organization. Cambridge University Press.

Lewis, A. H., Niznikiewicz, M. A., Delamater, A. R., \& Delgado, M. R. (2013). Avoidance-based human Pavlovianto-instrumental transfer. European Journal of Neuroscience, 38(12), $3740-$

3748. https://doi.org/10.1111/ejn. $\underline{12377 .}$.

Lin, Y., McKeachie, W. J., \& Kim, Y. C. (2003). College student intrinsic and/or extrinsic motivation and learning. Learning and Individual Differences, 13(3),

251-258. 
https://doi.org/10.1016/s10416080(02)00092-4.

Logan, F. A. (1968). Incentive theory and changes in reward. In Spence, K., W., \&, Spence, J., T. (Eds.), The psychology of learning and motivation (pp.1-30). New York: Academic Press. https://doi.org/10.1016/s00797421(08)60420-x.

López, M., \& Cantora, R. (2005). Condicionamiento, emoción y motivación. Aprendizaje de incentivo y regulación emocional de la conducta. Revista Electrónica de Motivación y Emoción, 8, 1-38.

Lorenz, K., \& Leyhausen, P. (1973). Motivation of human and animal behavior. an ethological view. Van Nostrand-Reinhold.

Lorrain, D., Arnold, G., \& Vezina, P. (2000). Previous exposure to amphetamine increases incentive to obtain the drug: long-lasting effects revealed by the progressive ratio schedule. Behavioural Brain Research, 107(1-2), 9-19. https://doi.org/10.1016/s01664328(99)00109-6.

Lovibond, P. F. (1983). Facilitation of instrumental behavior by a Pavlovian appetitive conditioned stimulus. Journal of Experimental Psychology: Animal Behavior Processes, 9(3), 225-247. https://doi.org/10.1037/00977403.9.3.225.
Mackintosh, N. J. (1988). The psychology of animal learning. Academic Press.

Mahler, S. V., Smith, K. S., \& Berridge, K. C. (2007). Endocannabinoid hedonic hotspot for sensory pleasure: anandamide in nucleus accumbens shell enhances 'liking' of a sweet reward. Neuropsychopharmacology, 32(11), 2267-2278. https://doi.org/10.1038/sj.npp.13 01376.

Marx, M. H., Henderson, R. L., \& Roberts, C. L. (1955). Positive reinforcement of the barpressing response by a light stimulus following dark operant pretests with no after effect. Journal of Comparative and Physiological Psychology, 48(2), 73-76. https://doi.org/10.1037/h004506 $\underline{2}$

Maslow, A. H. (1943). A theory of human motivation. Psychological Review, 50(4),

370-396. https://doi.org/10.1037/h005434 6.

Maslow, A. H. (1954). Motivation and personality. Harper.

Meyers, R., Pignault, A., \& Houssemand, C. (2013). The role of motivation and self-regulation in dropping out of school. Procedia - Social and Behavioral Sciences, 89, 270-275. https://doi.org/10.1016/j.sbspro.2 013.08.845.

Moon, L. E., \& Lodahl, T. M. (1956). The reinforcing effect of changes in illumination on lever-pressing in 
the monkey. The American journal of psychology, 69(2), 288-290.

https://doi.org/10.2307/1418162.

Morales Domínguez, J. F., \& Gaviria Stewart, E. (1990). La Motivación social. En S. Palafox y J. Vila. Motivación y emoción (pp. 147-195). Alhambra Longman.

Myers, A. K., \& Miller, N. E. (1954). Failure to find a learned drive based on hunger; evidence for learning motivated by "exploration". Journal of Comparative and Physiological Psychology, 47(6), 428436. https://doi.org/10.1037/h00 62664.

Parker, L. (1994). Rewarding drugs produce taste avoidance, but not taste aversion. Neuroscience and Biobehavioral Reviews, 19(1), 143151.

https://doi.org/10.1016/01497634(94)00028-Y.

Pavlov, I. P. (1927). Conditioned reflexes: an investigation of the physiological activity of the cerebral cortex. Oxford University Press.

Pavlov, I. P. (1932). The reply of a physiologist to psychologists. Psychological Review, $39(2)$, 91-127. https://doi.org/10.1037/h006992 $\underline{9}$.

Pecina, S., Smith, K. S., \& Berridge, K. C. (2006). Hedonic hot spots in the brain. The Neuroscientist, 12(6), 500-511. https://doi.org/10.1177/1073858 406293154.

Peciña, S., \& Berridge, K.C. (2005). Hedonic hot spot in nucleus accumbens shell: Where do $\mu$-opioids cause increased hedonic impact of sweetness? The Journal of Neuroscience, 25, 11777-11786. https://doi.org/10.1523/JNEUROS CI.4458-13.2014.

Peciña, S., \& Berridge, K. C. (1996). Brainstem mediates diazepam enhancement of palatability and feeding: microinjections into fourth ventricle versus lateral ventricle. Brain Research, 727(1-2), 2230. https://doi.org/10.1016/00068993(96)00325-3.

Peciña, S., Berridge, K. C., \& Parker, L. A. (1997). Pimozide does not shift palatability: separation of anhedonia from sensorimotor suppression by taste reactivity. Pharmacology Biochemistry and Behavior, 58(3), 801-811.

https://doi.org/10.1016/S00913057(97)00044-0.

Peterson, G. B., Ackilt, J. E., Frommer, G. P., \& Hearst, E. S. (1972). Conditioned approach and contact behavior toward signals for food or brainstimulation reinforcement. Science, 177, 10091011.

https://doi.org/10.1126/science.1 77.4053.1009. 
Petrovich, G. D., Ross, C. A., Gallagher, M., \& Holland, P. C. (2007). Learned contextual cue potentiates eating in rats. Physiology \& Behavior, 90(2-3), 362-

367. https://doi.org/10.1016/j.phy sbeh.2006.09.031.

Pfaffmann, C., \& Norgren, R. (1977). Sensory affect and motivation. Annals of the New York Academy of Sciences, 290(1), 18-34.

https://doi.org/10.1111/i.17496632.1977.tb39713.x.

Rana, S. A., \& Parker, L. A. (2008). Differential effects of neurotoxininduced lesions of the basolateral amygdala and central nucleus of the amygdala on lithium-induced conditioned disgust reactions and conditioned taste avoidance. Behavioural Brain Research, 189, 284-297.

https://doi.org/10.1016/j.bbr.200 8.01.005.

Real Academia Española. (2019). Diccionario de la lengua española (22.a ed.). Consultado en https://dle.rae.es/motivo\#B6vh6H f

Reeve, J. (2003), Motivación y emoción. McGraw-Hill.

Rescorla, R. A. (1988). Pavlovian conditioning: It's not what you think it is. American psychologist, 43(3), 151-160.

https://doi.org/10.1037/0003066X.43.3.151.
Reynolds, S., \& Berridge, K.C. (2002). Positive and negative motivation in nucleus accumbens shell: Bivalent rostrocaudal gradients for GABAelicited eating, taste "liking" / "disliking" reactions, place preference/avoidance, and fear. The Journal of Neuroscience, 22, 73087320. https://doi.org/20026734.

Richardson, D. K., Reynolds, S. M., Cooper, S. J., \& Berridge, K. C. (2005). Endogenous opioids are necessary for benzodiazepine palatability enhancement: Naltrexone blocks diazepam-induced increase of sucrose-liking. Pharmacology, Biochemistry and Behavior, 81, 657663.

https://doi.org/10.1016/j.pbb.200 5.05.006.

Robinson, J. S. (1961). The reinforcing effects of response-contingent light increment and decrement in hooded rats. Journal of Comparative and Physiological Psychology, 54(4), 470

473. https://doi.org/10.1037/h00 $\underline{47984}$

Robinson, T. E., Yager, L. M., Cogan, E. S., \& Saunders, B. T. (2014). On the motivational properties of reward cues: Individual differences. Neuropharmacology, $\quad 76, \quad 450-$ 459. https://doi.org/10.1016/j.neu ropharm.2013.05.040.

Rogers, P. J. (1990). Why a palatability construct is needed. Appetite, 14(3), 167-170. 
https://doi.org/10.1016/01956663(90)90083-K.

Ryan, R. M., \& Deci, E. L. (2000). Intrinsic and extrinsic motivations: Classic definitions and new directions. Contemporary Educational Psychology, 25(1), 54-67. https://doi.org/10.1006/ceps.199 9.1020.

Saunders, B. T., Yager, L. M., \& Robinson, T. E. (2013). Cue-evoked cocaine "craving": role of dopamine in the accumbens core. Journal of Neuroscience, 33(35), 1398914000.

https://doi.org/10.1523/jneurosci. 0450-13.2013.

Schmaltz, L. W., \& Theios, J. (1972). Acquisition and extinction of a classically conditioned response in hippocampectomized rabbits (Oryctolagus cuniculus). Journal of Comparative and Physiological Psychology, 79, 328-333.

Serafini, M., \& Cuenya, L. (2017). Evaluación del efecto del alojamiento en dos modelos animales para el estudio de las respuestas de frustración. Anuario de Investigaciones, 24, 311-318.

Shaham, Y., Shalev, U., Lu, L., de Wit, H., \& Stewart, J. (2002). The reinstatement model of drug relapse: history, methodology and major findings. Psychopharmacology, 168(1-2), 320. https://doi.org/10.1007/s0021 3-002-1224-x.
Smith, K. S., \& Berridge, K. C. (2005). The ventral pallidum and hedonic reward: Neurochemical maps of sucrose "liking" and food intake. Journal of Neuroscience, 25(38), 8637-8649.

https://doi.org/10.1523/JNEUROS CI.1902-05.2005.

Smith, K. S., \& Berridge, K. C. (2007). Opioid limbic circuit for reward: Interaction between hedonic hot spots of nucleus accumbens and ventral pallidum. Journal of Neuroscience, 27(7), 1594-1605. https://doi.org/10.1523/JNEUROS CI.4205-06.2007.

Smith, K. S. Berridge, K. C., \& Aldridge, J. W. (2011). Disentangling pleasure from incentive salience and learning signals in brain reward circuitry. Proceedings of the National Academy of Sciences, 108(27), 255-264. https://doi.org/10.1073/pnas.110 1920108.

Smith, K. S., \& Berridge, K. C. (2005). The ventral pallidum and hedonic reward: neurochemical maps of sucrose "liking" and food intake. The Journal of Neuroscience, 25, 86378649.

https://doi.org/10.1523/JNEUROS CI.1902-05.2005.

Söderpalm, A. H., \& Berridge, K. C. (2000). The hedonic impact and intake of food are increased by midazolam microinjection in the parabrachial nucleus. Brain Research, 877(2), 288- 
297. https://doi.org/10.1016/s000 6-8993(00)02691-3.

Solomon, P. R., \& Moore, J. W. (1975). Latent inhibition and stimulus generalization of the classically conditioned nictitating membrane response in rabbits (Oryctolagus cuniculus) following hippocampal ablation. Journal of Comparative and Physiological Psychology, 89(10), $1192-$

1203. https://doi.org/10.1037/h0 077183.

Solomon, P. R., Pomerleau, D., Bennett, L., James, J., \& Morse, D. L. (1989). Acquisition of the classically conditioned eyeblink response in humans over the life span. Psychology and Aging, 4(1), 34-41.

https://doi.org/10.1037/08827974.4.1.34.

Steiner, J. E., Glaser, D., Hawilo, M. E., \& Berridge, K. C. (2001). Comparative expression of hedonic impact: affective reactions to taste by human infants and other primates. Neuroscience \& Biobehavioral Reviews, 25(1), 5374. https://doi.org/10.1016/s0149 -7634(00)00051-8.

Stelly, C. E., Haug, G. C., Fonzi, K. M., Garcia, M. A., Tritley, S. C., Magnon, A. P., ... \& Wanat, M. J. (2019). Pattern of dopamine signaling during aversive events predicts active avoidance learning. Proceedings of the National Academy of Sciences, 116(27), 13641-13650. https://doi.org/10.1073/pnas.190 4249116.

Suárez, A., Mustaca, A., Pautassi, R., \& Kamenetzky, G. (2014). Ontogeny of consummatory successive negative contrast in rats. Developmental Psychobiology, 56, 989-998. https://doi.org/10.1002/dev.2117 8.

Tantot, F., Parkes, S. L., Marchand, A. R., Boitard, C., Naneix, F., Layé, S., ... \& Ferreira, G. (2017). The effect of high-fat diet consumption on appetitive instrumental behavior in rats. Appetite, 108, 203-211. https://doi.org/10.1016/j.appet.20 $\underline{16.10 .001 .}$.

Taormina, R. J., \& Gao, J. H. (2013). Maslow and the motivation hierarchy: Measuring satisfaction of the needs. The American journal of psychology, 126(2), 155-177. https://doi.org/10.5406/ameripsy c.126.2.0155.

Tindell, A. J., Smith, K. S., Peciña, S., Berridge, K. C., \& Aldridge, J.W. (2006). Ventral pallidum firing codes hedonic reward: When a bad taste turns good. Journal of Neurophysiology, 96, 2399-2409. https://doi.org/10.1152/in.00576. $\underline{2006 .}$.

Wahba, M. A., \& Bridwell, L. G. (1976). Maslow reconsidered: A review of research on the need hierarchy theory. Organizational Behavior and Human Performance, 15(2), 
240. https://doi.org/10.1016/003 0-5073(76)90038-6.

Weingarten, H. P. (1983). Conditioned cues elicit feeding in sated rats: A role for learning in meal initiation. Science, 220(4595), 431-433. https://doi.org/10.1126/science.6 836286.

Williams, B. A. \& Dunn, R. (1991). Preference for conditioned reinforcement. Journal of the Experimental Analysis of Behavior, 55(1), 37-46. DOI: 10.1901/jeab.1991.55-37

Winston, C. N., Maher, H., \& Easvaradoss, V. (2017). Needs and values: An exploration. The Humanistic Psychologist, 45(3), 295-311. https://doi.org/10.1037/hum0000 $\underline{054}$.
Wyvell, C. L., \& Berridge, K. C. (2000). Intraaccumbens amphetamine increases the conditioned incentive salience of sucrose reward: Enhancement of reward "wanting" without enhanced "liking" or response reinforcement. The Journal of Neuroscience, 20(21), 81228130. https://doi.org/10.1523/ine urosci.20-21-08122.2000.

Wyvell, C. L., \& Berridge, K. C. (2001). Incentive-sensitization by previous amphetamine exposure: Increased cue-triggered "wanting" for sucrose reward. Journal of Neuroscience, 21(19), pp. 7831-7840. https://doi.org/10.1523/INEUROS CI.21-19-07831.2001.. 